\section{El análisis estratigráfico constructivo* como estudio previo al proyecto de restauración arquitectónica: metodología y aplicación}

\author{
Camilla Mileto, Fernando Vegas \\ Universidad Politécnica de Valencia
}

\begin{abstract}
Resumen
En la primera parte de este texto, se presentan los fundamentos metodológicos que los autores utilizan en la aplicación del análisis estratigráfico constructivo. En los últimos años, diversos investigadores italianos y españoles han desarrollado la metodología del análisis estratigráfico constructivo aplicado a la arquitectura. Los autores del texto siguen las líneas generales de esta metodología intentando desarrollar por su parte una serie de aplicaciones y adaptaciones específicas según las exigencias de los casos estudiados.

En la segunda parte del texto, se presenta un caso de aplicación del análisis estratigráfico constructivo, el estudio de la iglesia de San Pedro en la Pobla de Benifassá. Se trata de un caso de un estudio general y completo del edificio que puede ilustrar correctamente la metodología general utilizada por los autores. Además, el caso permite reflexionar sobre el valor del análisis estratigráfico constructivo en relación con el estudio histórico-arquitectónico de un edificio.
\end{abstract}

Palabras clave: estratigrafía, metodología, arquitectura, estudio previo, proyecto de restauración

\begin{abstract}
The first part of this text presents the methodological foundations used by the authors in the application of stratigraphic wall analysis. In recent years, several Italian and Spanish researchers have developed the methodology of stratigraphic wall analysis applied to archaeology. The authors of this text follow the general lines of this methodology in an attempt to develop for themselves a series of specific applications and adaptations according to the demands of the cases studied.

The second part of the text presents a case of application of stratigraphic wall analysis in the study of the church of San Pedro in Pobla de Benifassá. This is a general, full study of the building which can correctly illustrate the general methodology used by the authors. Furthermore, this case allows us to reflect on the value of stratigraphic wall analysis in relation to the historic-architectonic study of a building.
\end{abstract}

Key words: stratigraphy, methodology, architecture, preliminary study, restoration project.

* Se propone utilizar el término análisis estratigrafico constructivo ya que se trata de un análisis, no solamente de una lectura (CABALLERO ZOREDA, 1995), basada en el método estratigráfico utilizado en arqueología, y que, para su correcta aplicación a la arquitectura, necesita de un conocimiento específico de los procesos constructivos ligados al desarrollo de una obra, al contexto cultural, y a las técnicas constructivas locales (DoGLIONI, 1997b).

\section{LA APLICACIÓN DEL MÉTODO DEL ANÁLISIS ESTRATIGRÁFICO CONSTRUCTIVO COMO ESTUDIO PREVIO AL PROYECTO DE RESTAURACIÓN ARQUITECTÓNICA}

Desde hace algunos años el método del estudio estratigráfico aplicado a los edificios históricos ha empezado a aparecer en los foros nacionales e internacionales de la restauración arquitectónica. Se trata de un método de análisis de la estratificación nacido en el mundo de la arqueología y que, al principio, se aplicó predominantemente a la estratificación arqueológica. El momento clave de la sistematización de este método está constituido, sin duda, por el trabajo de Edward C. Harris que, en 1979, publica sus «Principios de Estratigrafía Arqueológica». Sin embargo, las primeras aplicaciones de este método a la arquitectura se remontan sólo a principios de los años ochenta, cuando se empezó a aplicar como medio de conocimiento de la materialidad de la fábrica.

El objetivo principal del análisis estratigráfico murario es la lectura, documentación e interpretación de las diferentes fases constructivas del documento construido, pero existen una serie de objetivos secundarios que resultan de gran importancia cuando se aplica el método en el ámbito de un estudio previo a un proyecto de restauración. En primer lugar, la aplicación del método conlleva el estudio y documentación de los materiales y de las técnicas constructivas que componen el edificio, proporcionando un minucioso y atento conocimiento de la materialidad del mismo, objeto de la restauración. En segundo lugar, el conocimiento aportado por el método permite redactar un proyecto de restauración que, atento a los datos estratigráficos, apueste para la conservación de la materialidad, reduciendo la pérdida o la eliminación de los datos mismos.

Además, este método, gracias a su organización para la recogida de la información, permite ordenar y jerarquizar datos provenientes de otros tipos de estudios, convirtiéndose en un punto de enlace de diferentes estudios previos (estudio de materiales y técnicas constructivas, análisis de caracterización de los materiales, estudio de la degradación material, etc.)

\subsection{La investigación que se está desarrollando}

Desde hace algunos años, el grupo formado por los autores de este texto aplica el análisis estratigráfico constructivo a los edificios históricos, sobre todo, en el ámbito del estudio previo y del proyecto de restauración. Desde el principio, se han intentado investigar las posibilidades que el mismo método ofrece y, en particular, su capacidad de aplicación y de adaptación a un gran espectro de situaciones diferentes. En realidad, no se puede olvidar que, en la restauración arquitectónica, cada edificio representa un 
caso completamente singular y absolutamente diferente de todos los demás, de manera que, evidentemente, tanto su estudio como el proyecto para la intervención en el mismo, tendrán una importante especificidad. No se trata de trabajar sin una metodología precisa y clara de manera vaga e improvisada, sino de adaptar la metodología de estudio al caso específico del edificio que se debe estudiar, así como cada proyecto tendrá en cuenta los problemas de cada edificio.

En este sentido el método estratigráfico permite su adaptación a diferentes situaciones, dentro de un marco bien preciso y riguroso. Se trata de respetar todos los principios y el rigor de las fases de estudio, pudiendo, sin embargo, adaptar el método sobre todo en la cantidad (no en la calidad) de la información recogida, en el tipo de soporte gráfico o fotográfico, en el tipo de fichas de documentación de los datos, y en el tipo de interpretación de los datos (hipótesis de diferentes secuencias de periodos constructivos).

\subsection{Los casos de estudio presentados}

Los casos de estudio que se presentan brevemente constituyen el fruto de la aplicación del método del análisis constructivo por parte de los autores en los últimos años. Se trata de casos completamente diferentes entre ellos, tanto en lo que atañe al tipo de edificio estudiado (desde un amplio conjunto, hasta pequeños edificios o parte de ellos), como en las características que rodearon a dichos estudios (desde la posibilidad de confrontación con una amplia documentación histórica, hasta la total ausencia de noticias sobre los avatares del edificio), pasando por los objetivos (desde la investigación histórico-constructiva neta, hasta el estudio como parte de un estudio previo o como guía de un proyecto de restauración).

Se trata por tanto de un muestrario de situaciones que intenta reflejar la flexibilidad del método estratigráfico, ilustrando no solamente la metodología de aplicación, sino también reflexionando sobre una serie de cuestiones: desde la relación entre estratigrafía e historia de la arquitectura, hasta el papel que el análisis estratigráfico puede asumir en un estudio interdisciplinar o en un proyecto de restauración. Se demuestra igualmente la capacidad de adaptación y la flexibilidad que posee este método, a la vista de su aplicación a casos tan diferentes, sin menoscabar el rigor y la corrección de cada uno de los estudios.

En algunos casos, se han ensayado opciones que se salen ligeramente de la estricta aplicación del método, con el objetivo de proporcionar una serie de interpretaciones histórico-constructivas que se han demostrado de ayuda en la elaboración de las hipótesis de periodos constructivos. En otros casos el análisis estratigráfico se ha podido complementar con una serie de otros estudios, o ha sido parte del desarrollo de un estudio más completo previo a la restauración del edificio.

\subsection{La metodología utilizada}

La aplicación del método del análisis estratigráfico 'a la arquitectura, como se ha comentado, es relativamente reciente. Sin embargo, en los últimos veinte años, diferentes investigadores se ha ocupado de su sistematización metodológica, entre ellos recordamos: Brogiolo, Parenti, Francovich, Mannoni, Doglioni, Caballero, etc. Aunque cada uno propone particularidades y matices, sin embargo parece bastante clara la existencia de principios comunes y directivas metodológicas.

Dentro de estas líneas comunes los autores de este artículo están aplicando el método al estudio de la arquitectura histórica, proponiendo, como se ha dicho anteriormente, algunas adaptaciones específicas según las necesidades de los casos estudiados.

La metodología que aplicamos, se basa en tres fases fundamentales: la observación de los datos (estratos, bordes, superficies, identificación de unidades estratigráficas murarias...); la documentación de los datos (a través de dibujos y fichas); la interpretación de los datos, con la creación de distintas secuencias estratigráficas e hipótesis interpretativas. Estas tres fases se intentan mantener rigurosamente separadas y consecutivas en aras a garantizar la mayor objetividad posible de la recogida de la información.

\section{La lectura de los datos in situ}

La lectura directa in situ se realiza fundamentalmente mediante la atenta observación de las fábricas que componen el edificio, analizando sobre todo las discontinuidades entre materiales y técnicas constructivas diferentes. Con la experiencia se ha visto la utilidad de emplear un soporte fotográfico (mosaico fotográfico) como base de documentación de los datos in situ. El soporte fotográfico de hecho refleja fielmente los datos existentes eliminando el riesgo del filtro interpretativo del dibujo. Sobre el soporte fotográfico se dibujan los bordes de las unidades estratigráficas constructivas (zonas homogéneas que presentan las mismas características y los mismos materiales y que muestran haber sido construidas durante una misma acción constructiva) y, en un segundo momento, se identifican las relaciones de contemporaneidad, anterioridad y posterioridad, legibles en los bordes de las unidades estratigráficas constructivas (UEC), anotándolas a través de la leyenda propuesta por el profesor Doglioni (Doglioni, 1997) que permite una rápida y clara toma de datos. 


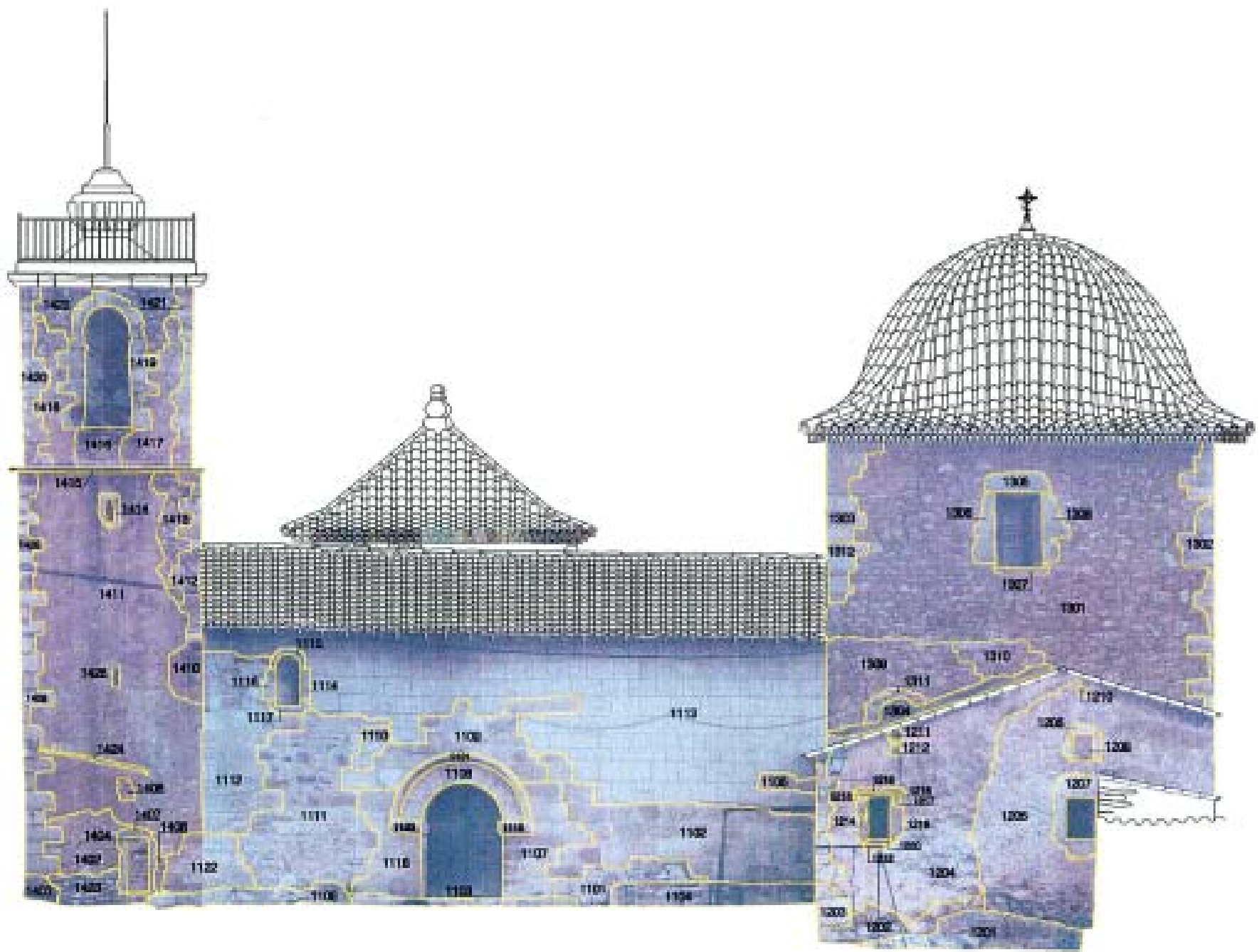

Fig. 1. Plano de análisis estratigráfico. Alzado Sur de la iglesia parroquial de San Pedro de la Pobla de Benifassà (Castellón)

\section{La documentación de los datos (Fig. 1)}

Durante la fase de documentación, se transcriben sobre el soporte preparado ex profeso (plantas, secciones y alzados) todas las observaciones realizadas mediante la lectura in situ, originando una serie de planos de análisis estratigráfico. En estos planos se dibujan los contornos de las diferentes masas murarias (planta y secciones) y de las unidades estratigráficas constructivas (alzados), estas últimas, numeradas con un número de identificación.

Habitualmente, para garantizar la máxima objetividad y legibilidad de los datos documentados, se utilizan dos tipos de soporte: por una parte, las plantas del edificio o del conjunto, realizadas mediante el levantamiento métrico, con un elevado nivel de detalle. Es importante tener en cuenta tanto el espesor de los muros, con todas las eventuales variaciones, como los eventuales cambios de dirección, esquinas, recovecos, etc., indicios de eventuales transformaciones de las fábricas. Por otra parte, se elabora un soporte gráfico-fotográfico de todos los alzados, tanto generales como de detalle, realizado a través de la rectificación de las fotografías ${ }^{1}$. Se considera de gran interés el uso de un soporte mixto gráfico y fotográfico ya que la fotografía aporta directamente una serie de datos que, en muchos casos, pueden resultar de gran importancia para la transmisión de las observaciones realizadas (color, textura, degradación, etc.) y que con un dibujo, por detallado que sea, difícilmente se pueden reflejar.

Con la experiencia se ha verificado la utilidad de completar los datos recogidos en los planos mediante fichas

\footnotetext{
1 Para la rectificación fotográfica se emplean software específicos en combinación con software de tratamiento de imágenes. Los puntos de apoyo necesarios para la rectificación fotográfica se obtienen con levantamiento topográfico. El montaje de las fotografías rectificadas en el levantamiento métrico se realiza con un software de dibujo asistido por ordenador.
} 

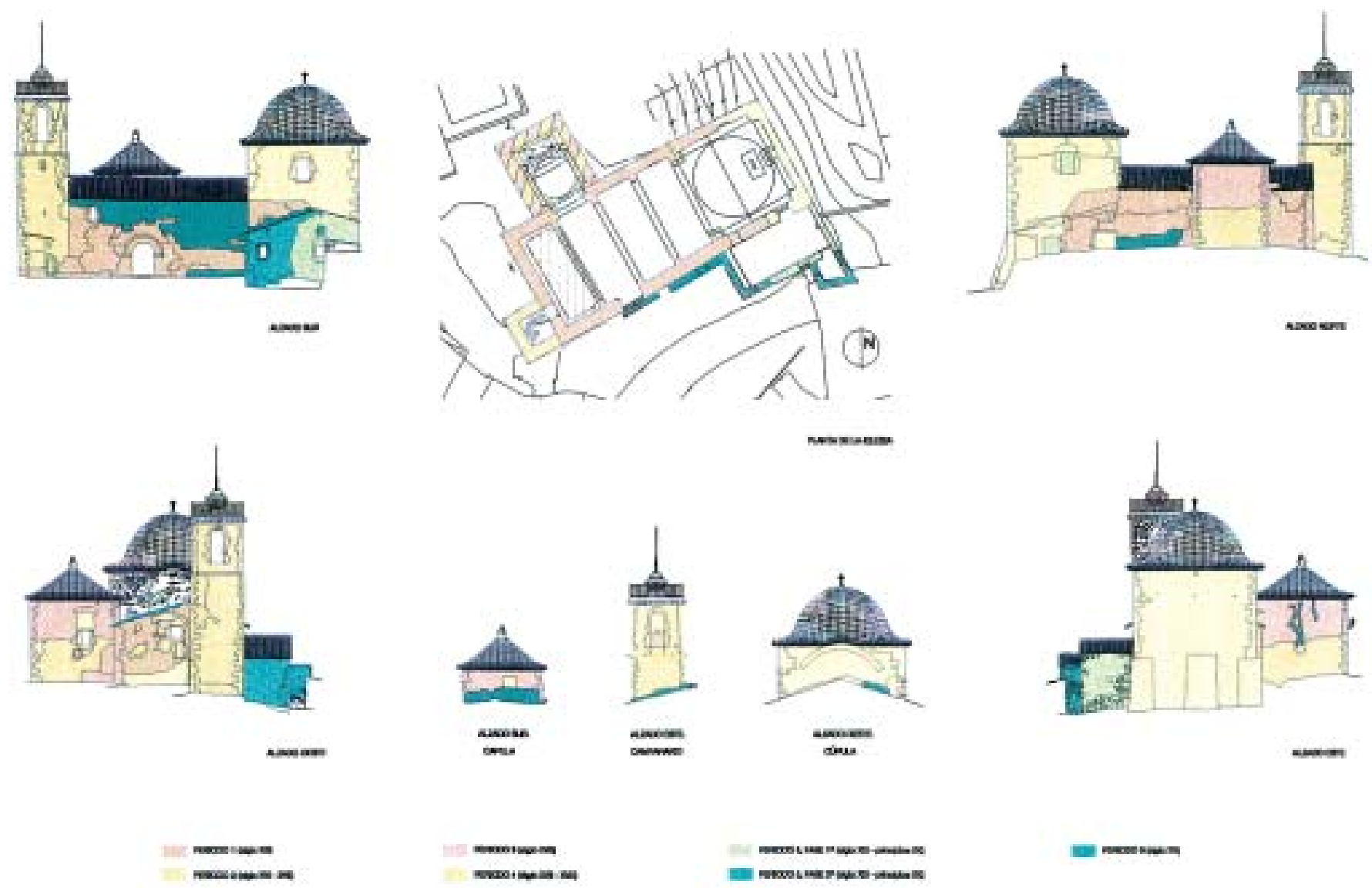

Fig. 2. Plano general de hipótesis de periodos constructivos de la iglesia parroquial de San Pedro de la Pobla de Benifassà (Castellón)

de análisis estratigráfico de tipo resumido. Se trata de una simplificación de las tradicionales fichas de análisis estratigráfico aplicadas en arqueología, según la línea propuesta por Brogiolo (1988). En las fichas se recoge el número de identificación de la unidad estratigráfica muraria, para conectar la localización en el plano con una descripción (materiales, técnicas utilizadas, posible función) y con las relaciones estratigráficas (de contemporaneidad —se une, igual a-, de anterioridad — cortado por, se le apoya, cubierto por, relleno por—y de posterioridad — corta, se apoya, cubre, rellena).

\section{La periodización y las hipótesis de periodos constructivos}

Una vez completada la lectura y la documentación de los datos parece oportuno pasar a la fase de interpretación de los mismos a través de su periodización, y sucesivamente a la elaboración de la hipótesis de la secuencia de los periodos constructivos del edificio.

Dada la cantidad de datos que se recogen en un estudio estratigráfico de un edificio y la complejidad tridimensional de la arquitectura, nos parece casi imposible o por lo menos notablemente arriesgado intentar realizar un diagrama de Harris, Harris Matrix, del edificio entero. Se ha ex- perimentado por tanto la aplicación de unos matrix limitados a sólo algunas partes donde existe una importante acumulación de datos, que se han denominado nudos de información. En esos puntos, resulta de gran interés y utilidad realizar unos pequeños matrix de detalle que, relacionados luego entre ellos, permiten la realización de la secuencia estratigráfica.

Una vez identificada la secuencia estratigráfica, gracias a la ayuda de la información histórica (investigación histórica documental) o de observaciones cronotipológicas, se puede intentar formular una hipótesis de periodos constructivos. Evidentemente, el nivel de detalle de la información histórica permite establecer hipótesis más o menos completas.

Igualmente, se hace necesario afirmar que las hipótesis elaboradas, a nuestro juicio, poseen el único objetivo del estudio de la sucesión de fases constructivas, que en ningún momento pretenden guiar las decisiones del proyecto. Es decir, la identificación de la evolución constructiva de los cuerpos que integran el conjunto no pretende utilizarse como excusa para una propuesta de intervención que menoscabe el valor de una fase en beneficio de otras. 
Para una mejor comunicación de las hipótesis de periodos constructivos formuladas resulta de gran conveniencia el empleo de una representación gráfica mediante esquemas de colores que facilita la rápida comprensión de la hipótesis. Eventualmente, además, se considera interesante proponer una visión general de la hipótesis de los diferentes periodos constructivos a través de una planta del conjunto con la representación de las masas murarias, tratadas con los mismos colores de los alzados para representar los diferentes periodos constructivos. (Fig. 2)

\section{UN EJEMPLO DE ANÁLISIS ESTRATIGRÁFICO CONSTRUCTIVO DE UN PEQUEÑO EDIFICIO MONUMENTAL: LA IGLESIA PARROQUIAL DE SAN PEDRO EN LA POBLA DE BENIFASSÁ (CASTELLÓN) ${ }^{2}$}

$\mathrm{El}$ análisis estratigráfico constructivo de la Iglesia de San Pedro se redactó en el ámbito del estudio previo al proyecto de restauración del edificio. El análisis estratigráfico, por tanto, se consideró no sólo como el medio para la identificación de los periodos constructivos del edificio, sino también para el registro de los datos materiales y de las relaciones entre ellos, con la meta de constituir una guía útil, de materiales, técnicas constructiva y huellas históricas presentes en la actualidad, para una redacción más consciente de los sucesivos proyectos de restauración.

\subsection{La Iglesia Parroquial de San Pedro en la Pobla de Benifassá}

La Pobla de Benifassá es el núcleo principal de un conjunto de asentamientos de pequeñas dimensiones que constituyen gran parte de la comarca de la Tinença de Benifassá, y se ubica arracimada en las faldas a oriente y mediodía de una colina entre el Barranco de la Pobla y el Barranquet de la Font. En la cima de esta colina, rodeada de un apretado anillo de casas adosadas abierto en el flanco norte, se encuentra ubicada la Iglesia Parroquial de San Pedro.

$\mathrm{El}$ acceso a la iglesia tiene lugar desde una plazoleta en el lado sur, vinculada al pueblo a través del anillo de casas alrededor de la misma, y el lado septentrional se encuentra abierto hacia la colina. La iglesia posee una única nave con cuatro tramos techados a dos aguas, separados por arcos perpiaños, y el cuerpo del altar cubierto por una cúpula. El último tramo a los pies de la nave se caracteriza por la presencia del coro y el acceso al campanario, adosado en el lado oriental de la iglesia, que aloja la escalera de subida al coro. El cuerpo del altar, de planta cuadrada, consiste en cuatro pilastras angulares y está cubierto por una cúpula, sobre pechinas bordeada de una sucesión de molduras. La Capilla de la Comunión se adosa a la nave en su fachada septentrional y presenta un tejado piramidal que cubre la cúpula interior de la capilla. En el lado sur, se adosa el cuerpo de la sacristía cubierto con tejado a dos aguas.

La iglesia es, sin dudas, en su configuración actual, el fruto de una serie de intervenciones, tanto de ampliación y añadido de nuevos cuerpos, como de transformación de las fábricas preexistentes.

\subsection{La metodología adoptada (Fig. 3)}

La iglesia de San Pedro constituye un pequeño edificio aislado que, como se ha descrito, es el fruto de una serie de intervenciones de remodelación y transformación. Por esta razón, el análisis estratigráfico se ha planteado a través del estudio principalmente de los cuatro frentes externos, alzados generales, a su vez divididos en alzados de detalle según la parte del alzado que se estaba analizando: por ejemplo en el frente sur (alzado general) se analizaron los alzados del campanario, de la nave, de la sacristía y del altar (alzados de detalle). En todos los alzados se identificaron las unidades estratigráficas.

El edificio presenta una estratificación legible sobre todo en el exterior, debido a que el interior ha sido enlucido y pintado en los últimos años. El estudio se dirige por ello principalmente a los paramentos exteriores, sin olvidar, sin embargo, la importancia de una lectura conjunta de las dos caras del muro. Por tanto, una vez identificadas las unidades estratigráficas en el exterior, y las relaciones entre ellas, se solapa el mapa de lectura estratigráfica tanto con la imagen de la cara interior del muro como con la planta, para comprobar posibles relaciones de interés. En particular en el caso de la capilla, este método ha dado la posibilidad de identificar la relación entre las diferentes unidades exteriores y las variaciones de la configuración interior.

Se acometió un estudio de caracterización ${ }^{3}$ de una serie de muestras de morteros y enlucidos pertenecientes al edificio, en primer lugar, con el objetivo de identificar de manera científica los materiales presentes, en aras de un futuro proyecto de restauración de las superficies de edificio, y en segundo lugar, con el ánimo de que estos ensayos pudieran confirmar o refutar las hipótesis que se han elaborado de sus periodos constructivos. Por ello, el estudio incluyó el

${ }^{2}$ El análisis estratigráfico de la Iglesia Parroquial de San Pedro de la Pobla de Benifassá ha sido realizado por los autores en el ámbito de un estudio previo más amplio, realizado por los mismos. El trabajo se ha realizado por encargo de la Consellería de Cultura y Ciencia de la Generalitat Valenciana en el año 2000. La infografía de los planos de levantamiento estratigráfico y de las hipótesis de periodos constructivos ha sido realizada por M. ${ }^{a}$ José Miñarro Viseras

${ }^{3}$ Los análisis de laboratorio han sido realizados por Francisco Martín Peinado, geólogo del Departamento de Edafología de la Universidad de Granada 


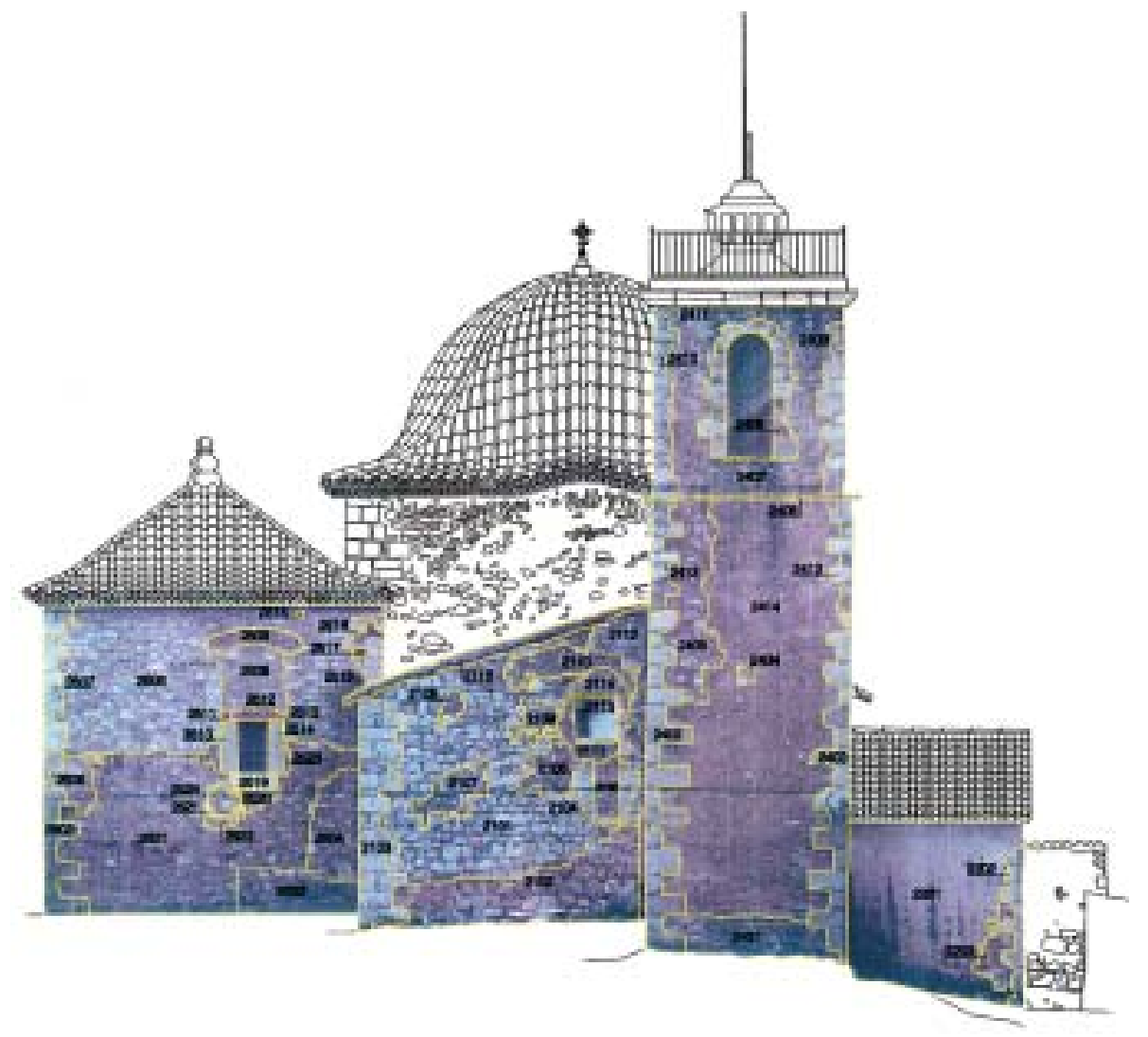

Fig. 3. Plano de análisis estratigráfico. Alzado Oeste de la iglesia parroquial de San Pedro de la Pobla de Benifassà (Castellón) análisis de once muestras con la técnica de la Difracción de rayos $\mathrm{X}$ a través de la determinación de carbonatos. Además, se acometió el estudio del estado de conservación del enlucido del campanario por microscopía de polarización. La analítica realizada no pretende ser un estudio exhaustivo de los materiales y su degradación, pero representa una primera aproximación a ellos en el marco de un estudio previo realizado en el ámbito de un proyecto de restauración de cubierta.

\subsection{Breve descripción de la hipótesis de los periodos constructivos de la iglesia}

Se han identificado seis periodos constructivos, elaborando una hipótesis de las fases de constructivas y de transformación sufridas por el edificio. La periodización, así realizada, se basa en los datos obtenibles actualmente a través de un estudio histórico indirecto realizado sobre fuentes documentales y fuentes bibliográficas.

\section{Periodo 1}

Se trata del periodo identificable con la iglesia primigenia, cuya fundación, se podría situar en la segunda mitad del siglo XIII. Actualmente, quedan de esta primera fábrica los cuatro tramos de la nave principal, caracterizados por cuatro arcos perpiaños, impostados sobre pilares de piedra cali- za con una escueta moldura como único capitel. A través del estudio estratigráfico del edificio, se han identificado una serie de elementos que indican la existencia del quinto tramo de la nave original, posteriormente manipulado con la construcción del cuerpo de la capilla mayor. Se detecta, de hecho, la presencia en el muro meridional, de una fábrica de mampostería que se diferencia de la fábrica principal y que se une a una fábrica de sillería y a un vano formado por sillería de piedra caliza. Esta fábrica constituiría el paramento del quinto tramo de la nave, y la fábrica de sillería formaría parte de la esquina de la antigua iglesia de reconquista. En segundo lugar, se detecta la presencia en el muro septentrional, en una puerta ahora tapiada, de una jamba de sillares de gran envergadura, que se entiende como el resto de la esquina de la primitiva iglesia. Cabe señalar que la superposición de la lectura estratigráfica de los alzados sur y norte con la planta del edificio, confirma la hipótesis de la existencia del quinto tramo.

\section{Periodo 2}

De este periodo no se encuentran noticias históricas claras, a excepción de algunas indicaciones. Por tanto, se ha prestado crédito a la observación material y a razonamientos cronotipológicos. Se trata de un largo periodo que podría abarcar los siglos XVI y XVII, según la observación de las ca- 


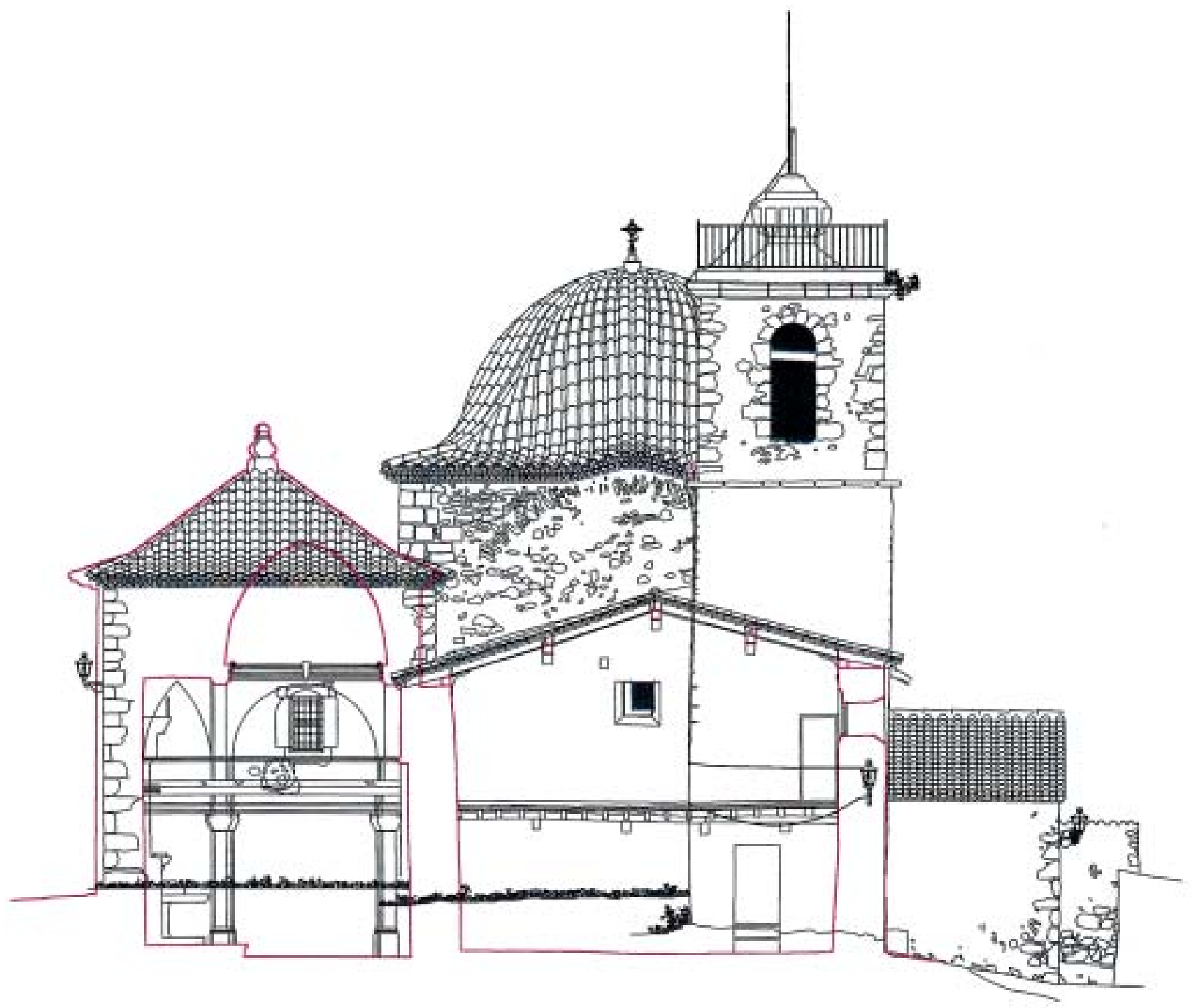

Fig. 4. Plano de superposición del alzado Este exterior con el correspondiente alzado interior de la iglesia parroquial de San Pedro de la Pobla de Benifassà (Castellón)

racterísticas de las fábricas que se identifican como pertenecientes a este lapso temporal. A este periodo, se adscribe el campanario en toda su dimensión ya que se trata de un cuerpo añadido a la primitiva iglesia. El otro elemento que parece ser identificable como perteneciente a este periodo es la parte inferior de la capilla de la Comunión.

\section{Periodo 3}

Como en el caso del periodo precedente, también en este caso no se posee ninguna noticia histórica. Simplemente, se observa la posibilidad de la existencia de una segunda fase de construcción de la capilla de la Comunión, probablemente muy próxima a la fase de su primera construcción, quizás en el intento de adaptar una primera pequeña capilla a una verdadera capilla de la Comunión. Esta fábrica se caracteriza por la presencia de dos vanos, uno en el alzado Oeste hoy tapiado y uno en el lado Sur también tapiado. A través de la superposición entre la cara exterior y la interior del muro (Fig. 4) se observa la absoluta incompatibilidad entre estas dos ventanas y la cúpula hoy existente. Por tanto, se deduce la existencia de, al menos, tres fases constructivas de la capilla.

\section{Periodo 4}

Se trata del periodo (posiblemente entre la segunda mitad del siglo XVII y principios del siglo XVIII) que contempla la anexión del cuerpo de la capilla mayor, con la absorción del quinto tramo de la nave para trasformarlo en un cuerpo 
cuadrado en el que se asienta la gran cúpula. Al mismo tiempo que se añade este cuerpo se realiza su decoración interior, con pilares, capiteles y entablamentos neoclásicos. Probablemente, al mismo tiempo que se construye la capilla mayor, se transforma una vez más la capilla de la comunión para que adquiera un aspecto más parecido a la nueva transformación.

\section{Periodo 5}

Al quinto periodo (entre el siglo XIX y principios del XX) pertenecen dos fases constructivas difícilmente datables en el tiempo con una mayor aproximación, debido a su escasa caracterización estilística. Se trata, en una primera fase, de la construcción de un añadido en el lado meridional del cuerpo de la capilla mayor.

\section{Periodo 6}

Corresponde a la segunda mitad del siglo XX, aproximadamente, de la Guerra Civil hasta nuestros días. A este periodo pertenecen, fundamentalmente, las obras de la inmediata posguerra, en que se interviene cubriendo en parte la fábrica de la fachada sur con un enlucido de cemento y, probablemente, reparando la cubierta que parece, según fuentes populares, haber sido objeto de un incendio durante la guerra.

\subsection{La relación entre el análisis estratigráfico constructivo y la investigación histórica documental}

En el caso de la Iglesia Parroquial de San Pedro en la Pobla de Benifassá la investigación histórica apenas pudo proporcionar información ${ }^{4}$. Existían fundamentalmente una serie de hipótesis tipológicas basadas en la observación de edificios similares. Mediante estas comparaciones se había elaborado una hipótesis sobre la existencia de un quinto tramo de la nave de la iglesia. Se debe destacar cómo esta hipótesis fue completamente confirmada gracias a la lectura directa de las fábricas que además desveló el proceso completo de ampliación de la cabecera de la iglesia. Además se debe indicar la absoluta inexistencia de información sobre la construcción y sucesivas transformaciones de la capilla de la Comunión, mientras que el análisis estratigráfico murario pudo desvelar la compleja evolución.
El caso de la iglesia de San Pedro se propone sólo como una excusa para poder reflexionar sobre la relación entre análisis estratigráfico constructivo y estudio histórico documental. Se estima que las dos aproximaciones son totalmente complementarias y necesarias, no pudiendo considerar una más importante que la otra. El estudio histórico documental proporciona información ligada al proceso histórico y a la cultura del momento y, en algunos casos, indica unas fechas históricas precisas. El análisis estratigráfico constructivo en cambio proporciona un conocimiento detallado de la materialidad del edificio y de las huellas de sus transformaciones. Es por tanto absolutamente necesario considerar estas dos aproximaciones como complementarias y necesarias para el correcto conocimiento del edificio, tanto en aras de un conocimiento histórico en sí mismo como de un conocimiento básico para la redacción de un proyecto de restauración.

\section{Bibliografía}

AA.VV., 1996-2001, Archeologia dell'architettura, números de I a VI, Florencia.

AA.VV., 1996, Arqueología de la arquitectura, actas del curso homónimo, Burgos.

AA.VV., 2002, Arqueología de la Arquitectura, n. ${ }^{\circ}$ I, Vitoria-Gasteiz.

AA.VV., 1990, Coloquio Hispano-italiano de Arqueología Medieval, Granada.

AA.VV., 1995, Leer el documento construido. Informes de la construcción, n. ${ }^{\circ}$ 435, Madrid.

Brogiolo G.P., 1988, Archeologia dell'edilizia storica, Como.

Brogiolo G.P., De Marchi P.M., Della Torre S., 1999, I metodi dell'archeologia e il progetto d'intervento sull'architettura, Como.

Caballero Zoreda L., 1995, Método para el análisis estratigráfico de construcciones históricas o Lectura de paramentos, en AA.VV., Leer el documento construido. Informes de la construcción, n. ${ }^{\circ}$ 435, págs. 37-38.

Doglioni F., 1997a, Conseguenze del restauro sulla stratificazione e contributi della stratigrafia al restauro, en AA.VV., Archeologia dell'architettura, n. ${ }^{\circ}$ II, Florencia, págs. 207-213.

Doglioni F., 1997b, Stratigrafia e restauro, Trieste.

HARRIS E.C., 1991, Principios de estratigrafía arqueológica, Barcelona.

Mileto C., 2000, Algunas reflexiones sobre el Análisis Estratigráfico Murario, en Loggia, n. ${ }^{\circ}$ 9, Valencia, págs. 80-93.

PARENTI R., 1997, Historia, importancia y aplicaciones del método de lectura de paramentos, en AA. VV., Teoría e historia de la restauración, Madrid, págs. 259-269.

TORSELlo B.P., 1989, Scrittura, segni e memoria, en Il Cantiere della conoscenza, il cantiere di restauro, actas del Convegno di Studi di Bressanone, Padua, págs. 663-670.

\footnotetext{
${ }^{4}$ Las noticias históricas se han extraído del trabajo de investigación de Arturo Zaragozá (Naves de arcos diafragma y armadura de madera en la arquitectura medieval valenciana») y de la memoria redactada para este estudio previo por los profesores Violeta Montoliú y David Navarro.
} 